
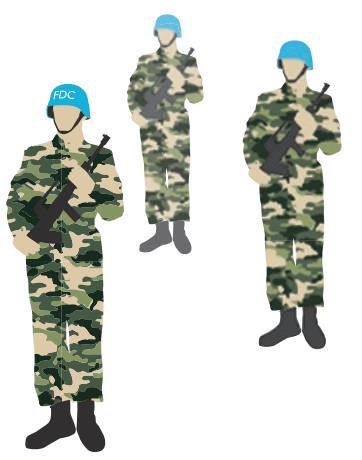

\title{
LYMPHOID FOLLICLES
}
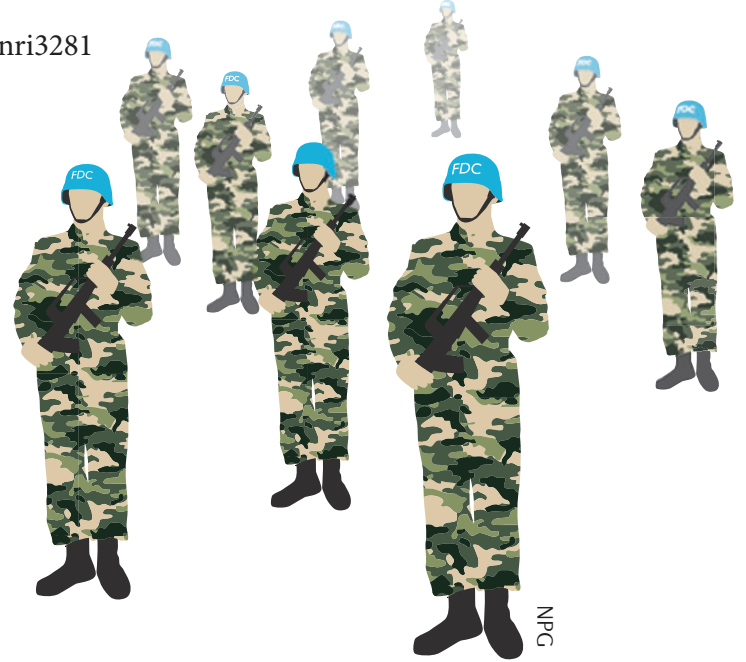

\section{FDCs - ubiquitous and ready for action}

Follicular dendritic cells (FDCs) trap immune complexes to engage $B$ cells that are trafficking through lymphoid follicles, including the tertiary lymphoid follicles that can arise anywhere in the body during chronic inflammation. The origin of FDCs has been unclear, but now Krautler and colleagues report in Cell that FDCs arise from ubiquitous perivascular cell precursors that express platelet-derived growth factor receptor- $\beta$ (PDGFR $\beta$ ).

Following the observation that some cells in the splenic marginal and $\mathrm{T}$ cell zones in mice express certain FDC markers - such as MFGE8 (also known as lactadherin) - but not the immune complex-trapping molecules that are characteristic of mature FDCs, Krautler et al. hypothesized that these cells were FDC precursors (pre-FDCs).

Previous studies have shown that tumour necrosis factor receptor 1 (TNFR1), lymphotoxin- $\beta$ receptor (LT $\beta R$ ) and $B$ cells expressing $L T \alpha \beta$ are required for the induction and maintenance of FDCs. Using mice lacking TNFR1 or LT $\beta R$, the researchers found that pre-FDC development in the spleen required LT $\beta R$ but not TNFR1 signalling. Furthermore, pre-FDCs were present in mice lacking $B$ and $T$ cells (Rag1 ${ }^{-/}$mice) but were reduced in number in mice lacking $B, T$ and natural killer (NK) cells (Rag2 ${ }^{-1-l} l 2 \mathrm{rg}^{-/-}$mice). This reduction was caused by the depletion of lymphoid tissue-inducer cells in these mice and not by NK cell depletion per se. When Krautler et al. induced follicle development in $\mathrm{Rag}^{-/-} \| \mathrm{l} 2 \mathrm{rg}^{-/-}$ mice by administering $\mathrm{Mfge}^{\mathrm{s}^{-/}}$bone marrow, they saw an influx of $B$ and $T$ cells at perivascular sites in the spleen. The perivascular areas then became populated by MFGE8 ${ }^{+}$(host-origin) pre-FDCs, suggesting that lymphocytes are sufficient to induce the expansion of the pre-FDC population.

These data prompted the researchers to ask whether pre-FDCs were mural cells - that is, vascular smooth muscle cells and pericytes that surround and maintain blood vessels. They found that MFGE8 $8^{+}$pre-FDCs, but not mature FDCs, express PDGFR $\beta$, as mural cells do. Conditional ablation of the mural cell lineage from mature spleens in mice led to a reduction in FDC networks and severely affected the overall organization of the B cell follicles. In addition, when PDGFR $\beta^{+}$stromal vascular cells were treated with agonistic LT $\beta R$-specific antibodies and TNF, mature FDC markers were induced and PDGFR $\beta^{+}$expression was lost, suggesting that PDGFR $\beta^{+}$cells in the vasculature are pre-FDCs.

But can pre-FDCs be found in all vascularized organs? Krautler et al. found that treating mice with agonistic LT $\beta R$-specific antibodies induced the generation of MFGE8 ${ }^{+}$cells in tertiary lymphoid tissues in the liver, kidney and white adipose tissue, sites normally devoid of FDCs. Furthermore, when kidneys from transgenic mice with renal LTa overexpression but no FDCs were transplanted into mice deficient for prion protein (which is highly expressed by FDCs), FDC networks re-formed in the transplanted kidneys. This confirmed that pre-FDCs exist in non-lymphoid organs. When the PDGFR $\beta^{+}$ stromal vascular cells were transplanted into LT $\beta$ R-deficient kidney capsules of FDC-deficient mice and were stimulated to induce the differentiation of FDCs, mature FDCs were generated that recruited $B$ cells and were capable of trapping immune complexes.

These results help to explain how tertiary lymphoid tissues develop rapidly at sites of chronic inflammation, and future research focusing on the steps by which PDGFR $\beta^{+}$ pre-FDCs differentiate into mature FDCs will further this understanding.

Ezzie Hutchinson

ORIGINAL RESEARCH PAPER Krautler, N. J. et al. Follicular dendritic cells emerge from ubiquitous perivascular precursors. Cell 150, 194-206 (2012) 